\title{
Optical service unit (OSU)-based next generation optical transport network (NG OTN) technology and verification
}

\author{
Lirong Bai* \\ China Mobile Communications Group Co., Ltd. Beijing 100078, China
}

\begin{abstract}
With the development of transmission technologies, optical transport network (OTN) has evolved from a digital encapsulation technology to a network technology that supports multi-service transport. As the OTN gradually expands to the edge of the metropolitan area network (MAN) and enterprise users have higher quality requirements on bandwidth and service security, more and more private line services are provided via OTN network. Therefore, the OTN container is transformed from an optical data unit (ODU) to an optical service unit (OSU). The OTN industry has studied several OSU technologies to fit the development trends of NG OTN, aiming at transporting $2 \mathrm{Mbit} / \mathrm{s}$ to $100 \mathrm{Gbit} / \mathrm{s}$ constant bit rate (CBR) services and Ethernet services with high efficiency and more flexibilities, as well as reducing OTN network deployment and operation and maintenance (O\&M) costs. In this work, several key technologies in NG OTN are introduced including NG OTN technical architecture, OSU frame format, client mapping and OSU multiplexing. Based on these technologies, we (China Mobile) took the lead in conducting the industry's first NG OTN test in Qingdao, which verified the service transport capability and lower latency performance of OSUs and promoted the maturity of the NG OTN industry chain.
\end{abstract}

\section{Background}

OTN consists of a set of powerful technologies that offer high-speed traffic transport, network management and supervisory, and service grooming and switching. OTN technologies and standards keep continuous innovation, and evolve from supporting 2.5 $\mathrm{Gb} / \mathrm{s}$ service since around year 2000 to beyond $100 \mathrm{~Gb} / \mathrm{s}$ recently [1, 2]. In addition to bit rate upgrade, OTN technologies are also being deployed from backbone network to metro network and even to metro edge, so that OTN equipments are getting closer and closer to end users while more and more new service demands and technical requirements are emerging.

With the rapid developments of enterprise services going on cloud and private lines, the requirements on service level agreement (SLA), secure and stable networking, and reasonable cost with fast service response are getting higher. To improve the efficiency and

${ }^{*}$ Corresponding author: bailirong@,chinamobile.com 
flexibility of OTN for carrying private line and cloud services, the optical communication industry has proposed the NG OTN technology based on OSU. This OSU-based technology can effectively carry private line services with high quality, and will meet different service transport requirements from various industry sectors like finance, energy, government, education, etc. In this work, we will first introduce the industry progress of the OSU-based NG OTN technology, and then introduce our proposed technical enhancement and the first technical verification of this technology.

\section{NG OTN technical characteristics}

For OTN deployed on the backbone network and the core level of the metropolitan area network, the current data mapping solution based on optical data unit (ODUk) can achieve good bandwidth utilization since the bandwidth span of the carried services is relatively small. However, this traditional solution that utilizing time slot interleaving will be unsustainable once OTN is deployed on the edge of the metropolitan area network where larger service bandwidth span is involved. Therefore, it demands technical innovation to develop more flexible service carrying method, e.g. OSU. OSU attached with tributary port number (TPN) can dynamically identifying the corresponding relationship between OSU and services. Moreover, as OSU is compatible with smaller service bandwidth, it can afford over 1000 ODU connections, while traditional OTN supports merely 80 connections [2]. In addition, it is demanded that the bandwidth upgrade is imperceptible in case of private lines application, so that OSU should be capable of switching bandwidth simply without affecting the services.

From the perspective of equipment, current OTN uses superimposed multiple boards to accomplish multi-service access. Essentially, the services collecting still rely on the synchronous digital hierarchy (SDH), packet transport network (PTN), or passive optical network (PON), followed by a simple stacking and combing of these techniques in the OTN equipment $[3,4]$. This simple multiple boards stacking method brings obstacle for network partitioning and management. Therefore, NG OTN demands high efficiency, simple and unified service carrying form, so as to reduce deployment and operation cost.

\section{NG OTN key technologies}

\subsection{Mapping client signals to OSU frames}

In order to efficiently carry services, service-oriented OSU is required and simultaneously, it should be compatible with the existing OTN architecture. The OSU container can efficiently carry services with minimum bit rate to a few Mbit/s. Therefore, services such as constant bit rate (CBR) services, packet services, and new services can be mapped into the OSU by using innovative adaptation technologies. As shown in Fig. 1, the OSU can be multiplexed into lower order ODUj and then multiplexed into higher order ODUk/ODUCn for transmission. Alternatively, the OSU can be directly multiplexed into higher order ODUk/ODUCn for transmission.

Currently, there are two OSU implementation solutions in the industry to solve the complexity and flexibility of carrying low-rate services and future services, including the 66b-based OSU solution and block-based OSU solution. 


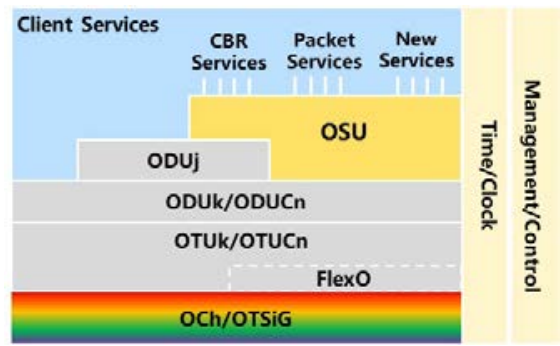

Fig. 1. NG OTN technique architecture.

\subsubsection{6b-based OSU solution}

The 66b-based OSU solution defines the OSU frame format based on 66b code block. An optical payload unit (OPU) payload area is divided into a plurality of 66-bit units, and an OSU frame format is constructed based on $\mathrm{N}$ of $66 \mathrm{~b}$ code blocks. Firstly, a client service data is transcoded into a $66 \mathrm{~b}$ code block stream, and then mapped into the payload area of an OSU frame by synchronous mapping procedure (e.g. bit-synchronous mapping procedure, BMP). The OSU overhead provides an end-to-end client service management and maintenance function. The quantity $\mathrm{N}$ is determined based on the carried client rate. The first $\mathrm{M}$ of $66 \mathrm{~b}$ code blocks are the overhead of OSU, and the remaining $(\mathrm{N}-\mathrm{M})$ of $66 \mathrm{~b}$ code blocks are the payload of OSU. Secondly, the OSU configuration information and multiple OSU frames with their clock information are multiplexed into the 66-bit units of ODUj. Finally, ODUj is multiplexed into a higher order ODUk, and is sent by an optical transport unit (OTU) or a flexible OTN interface (FlexO). In the case of 66b-based solution, the configuration information needs to be transported by the OPU payload area, and it also needs to allocate these $66 \mathrm{~b}$ code blocks by a Sigma-Delta mechanism according to their sequence.

\subsubsection{Block-based OSU solution}

The block-based OSU solution defines a 192-byte OSU frame structure. It uses n x $2 \mathrm{Mbit} / \mathrm{s}$ $(n \geqslant 1)$ bit rate to carry CBR services and packet services. There are two potential frame structures. Option 1 is a 192-byte single frame structure of OSU. It has a fixed overhead area and a payload area in each OSU single frame, where the overhead area includes an overhead and a CRC8 field, as shown in FIG. 2. Option 2 is a frame format consisted of $\mathrm{N}$ 192-byte blocks, where the first 192-byte block carries the overhead information, and the following N-1 192-byte blocks as the payload area carry services and partial overhead information such as automatic protection switching (APS) and clock frequency information. As option 1 inherits the traditional OTN thought and is easier to implement, it gets a majority of support currently.

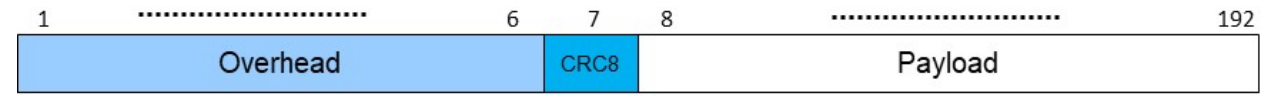

Fig. 2. OSU frame format.

When multiple OSUs are multiplexed into OPUs, each OSU frame needs to carry a TPN to identify the mapping relationship between OSU frames and tributary ports. The TPN must be unique at the server layer to ensure that the receiving side can correctly distinguish tributary port numbers. Its function is similar to the multiplexing structure identifier (MSI) function of OTN. 
The OPU payload area is divided into a plurality of 192-byte units, and each 192-byte unit is a payload block (PB). Multiple OSUs are multiplexed into the OPU payload area by the specific algorithm.

\subsection{Client signal mapping process}

There are two types of NG OTN services: CBR services and packet services. CBR services are mapped into OSUs via asynchronous mapping, as shown in Figure 3. The number of CBR services bytes which are carried in OSU should be determined firstly, and then these CBR services bytes are mapped into the OSU payload asynchronously, other locations in the payload should filled with the fixed stuff bytes. The Timestamp information and the byte quantity information (PLn) are carried in the OSU overhead bytes.

\begin{tabular}{|c|c|c|c|c|ccc|c|}
\hline Overhead & Timestamp & RES & PLn & CRC8 & Carrying CBR services & NS \\
\hline
\end{tabular}

Fig. 3. Mapping CBR services into OSUs.

Packet services are mapped into OSUs through $257 \mathrm{~b}$ transcoding, as shown in Figure 4. Firstly, the packet service should be extracted as MAC frames, and the MAC frames are encoded as $66 \mathrm{~b}$ and transcoded into $257 \mathrm{~b}$ codes. The $257 \mathrm{~b}$ code streams are mapped to the OSU payload area. The first control bit of $257 \mathrm{~b}$ and the start location information of $256 \mathrm{~b}$ (PTR) should be carried in the OSU overhead.

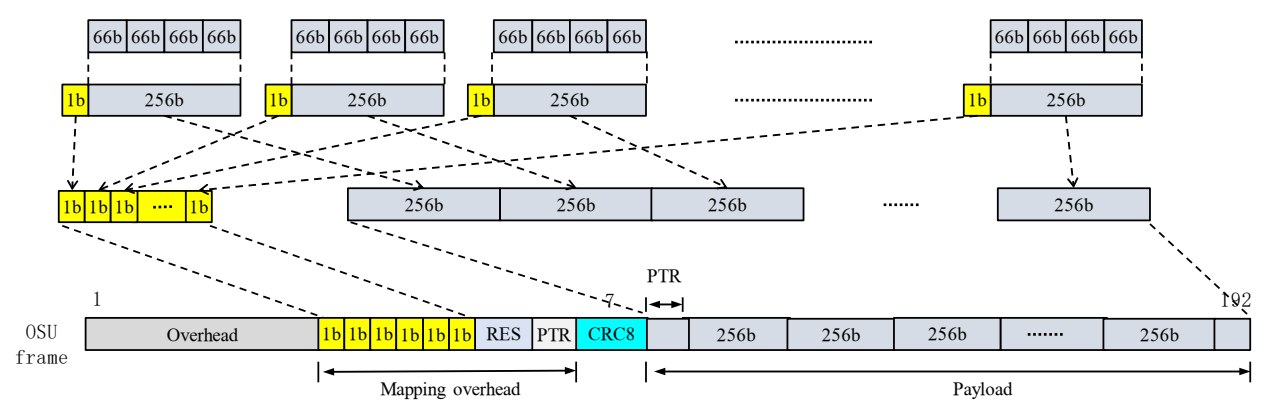

Fig. 4. Mapping packet services into OSUs.

Based on our proposed scheme together with the industry discussion, at the ITU-T SG15 plenary meeting in January 2020, ITU-T agreed to initiate a new G.osu project. The new G.osu clearly supports OSU service layer, switching and OSU bandwidth adjustment functions, and this new OSU should further map into OPU. Correspondingly, the China Communications Standards Association (CCSA) has initiated the corresponding standards work in China, and some of the work has been completed.

\section{NG OTN verification}

In January 2020, we (China Mobile) took the lead in conducting the industry's first NG OTN trial in Qingdao, China, focusing on small-granularity service carrying, ultra-low latency, and bandwidth adjustment. The trial system is shown in Fig. 5(a), which includes network elements for networking and testers for service testing. The test result shows that NG OTN supports lossless bandwidth adjustment from $2 \mathrm{Mbit} / \mathrm{s}$ to $100 \mathrm{Gbit} / \mathrm{s}$, with small- 
granularity service grooming at a step of $2 \mathrm{Mbit} / \mathrm{s}$, which effectively matches almost all service granularities such as plesiochronous digital hierarchy (PDH), SDH, and Ethernet (ETH). Moreover, compared with the currently widely-used Ethernet over SDH (EOS) technology, the NG OTN technology has significantly lower the end-to-end latency. As shown in Fig. 5(b), the latency for $2 \mathrm{Mb} / \mathrm{s}$ service is reduced from $4398 \mu$ s with EOS technology to $1289 \mu$ s with OSU-based NG OTN technology, and reduced from $726 \mu$ s to $471 \mu \mathrm{s}$ for the $100 \mathrm{Mb} / \mathrm{s}$ service. In addition to the end-to-end latency, the forwarding latency of a single node is reduced to $10 \mu \mathrm{s}$. In the future, we will continue to optimize NG OTN technologies and further verify the application effect of NG OTN in carrying integrated service scenarios such as government, enterprise private line and home broadband.

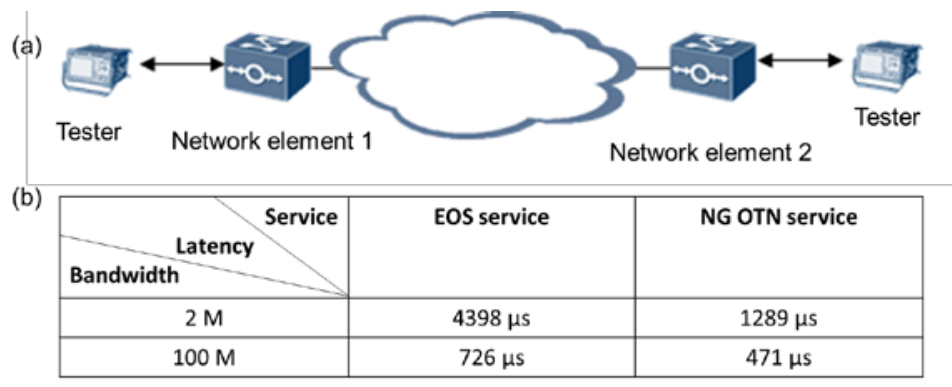

Fig. 5. The first NG OTN test. (a) Schematic of the test system; (b) Comparison of the latency performance between EOS service and NG OTN service for different bandwidth service.

\section{Conclusion}

As OTN networks are deployed on a large scale and are gradually moved down to the MAN and access network, OTN networks will gradually migrate to directly carry end-user services, transforming from pipe channels to service channels. This change will bring major innovations in OTN switching architecture, mapping, multiplexing solutions, and differentiated feature capabilities, further simplifying the network architecture and minimizing the capital expenditure (CAPEX) and operating expense (OPEX), which drives the evolution from OTN to NG OTN.

China Mobile and equipment manufacturers are leading the industry in the nextgeneration OTN research and have made great contributions to the standardization of NG OTN. With the gradual standardization of NG OTN, the related industry chain will become more mature and will be widely deployed and put into commercial use worldwide.

\section{References}

1. M. Carroll, J. Roese, T. Ohara, IEEE Commun. Mag. 48, 46 (2010)

2. S. S. Gorshe, J. Lightwave Technol. 36, 19 (2018)

3. Ministry of Industry and Information Technology of the People's Republic of China, China Telecommunication Industry Standard YD/T 1990-2019: General technical requirements for optical transport network (OTN) (2019)

4. Ministry of Industry and Information Technology of the People's Republic of China, China Telecommunication Industry Standard YD/T YD/T 2484-2020: Technical requirement on packet enhanced optical transport network (OTN) equipment (2020) 